\title{
Chronic Calcific Pancreatitis Associated Diabetes Presenting as Involuntary Weight Loss and Multiple Soft Tissue Calcifications in a Young Male
}

\author{
Ravi Kant ${ }^{1}$ Aditya Sudan ${ }^{2}$ \\ Associate Professor and Head ${ }^{1}$, Junior Resident ${ }^{2}$ \\ Division of Diabetes and Metabolism \\ Department of Medicine, AIIMS Rishikesh
}

\begin{abstract}
Introduction: Chronic Calcific Pancreatitis, a rare form of secondary diabetes occurs due to recurrent alcohol induced acute pancreatitis.

Case Summary: A 28 year old male patient presented with significant involuntary weight loss associated with a history of passing clay colored sticky stools for the past 1 year. He was also detected to have deranged blood sugars on routine work up at a local hospital for increased thirst and increased urine output. There was a history of recurrent bouts of moderate to severe abdominal pain over the past 3 to 4 years. At the time of initial presentation his Random blood sugar values was $468 \mathrm{mg} / \mathrm{dL}$. Other routine tests tests were within normal limits. His serum amylase levels were $185 \mathrm{U} / \mathrm{L}$ and serum Lipase levels were $467 \mathrm{U} / \mathrm{L}$.

Conclusions: Chronic fibro calcific pancreatitis is a rare cause of diabetes in young patients but has a characteristic clinical picture with a young patient presenting with features of malabsorption and low BMI, low propensity for DKA, low beta cell reserve and sensitivity to Insulin .
\end{abstract}

Key Words: FPCD-chronic fibrocalcific pancreatic disease, TFCP-tropical fibrocalcific pancreatitis, TIGAR -o-toxic, idiopathic, genetic, autoimmune-obstructive

\section{Introduction}

Chronic calcific pancreatitis is a rare form of secondary diabetes. ${ }^{1}$ Chronic calcific pan-creatitis most commonly occurs in adult patients due to recurrent alcohol induced acute pancreatitis. Tropical Fibro-calculus Pancreatitis is also an important cause in tropic coun-tries. In India the TFCP is most commonly seen in the south and east of the country, par-ticularly in Tamil Nadu and Kerala. Chronic pancreatitis and diabetes also occur in a rare form of Maturity Onset Diabetes of Young (MODY type 8) due to defect in the gene encoding cholesterol ester lipase.

Development of diabetes is a common complication of chronic pancreatitis and such pa-tients usually have significant weight loss due to the associated malabsorption. $^{2}$

These patients show very low serum C-peptide levels due to decreased pancreatic beta cell reserve and have very high sensitivity to insulin, with glycemic control being achieved at very low doses of insulin therapy .

\section{Case summary}

A 28 year old male patient presented with significant involuntary weight loss associated with a history of passing clay colored sticky stools for the past 1 year. He was also detect-ed to have deranged blood sugars on routine work up at a local hospital for increased thirst and increased urine output. There was a history of recurrent bouts of moderate to 
severe abdominal pain over the past 3 to 4 years.

There was no similar history in any first degree relatives. He had a history of only occa-sional alcohol intake and no history of binge drinking followed by onset of severe ab-dominal pain.

On examination he was conscious, oriented to time, place and person and was thinly built. His vital parameters were stable but his BMI was only 17.3. There was no pallor, icterus, cyanosis, clubbing or lymphadenopathy. Per abdomen was sound and there was a mass palpable just to the right of the midline in the epigastric and umbilical regions, which had a smooth surface, firm to hard consistency, did not move with respiration and became less prominent on contraction of the abdominal wall muscles. The respiratory, cardiovascular and neurological examination was within normal limits.

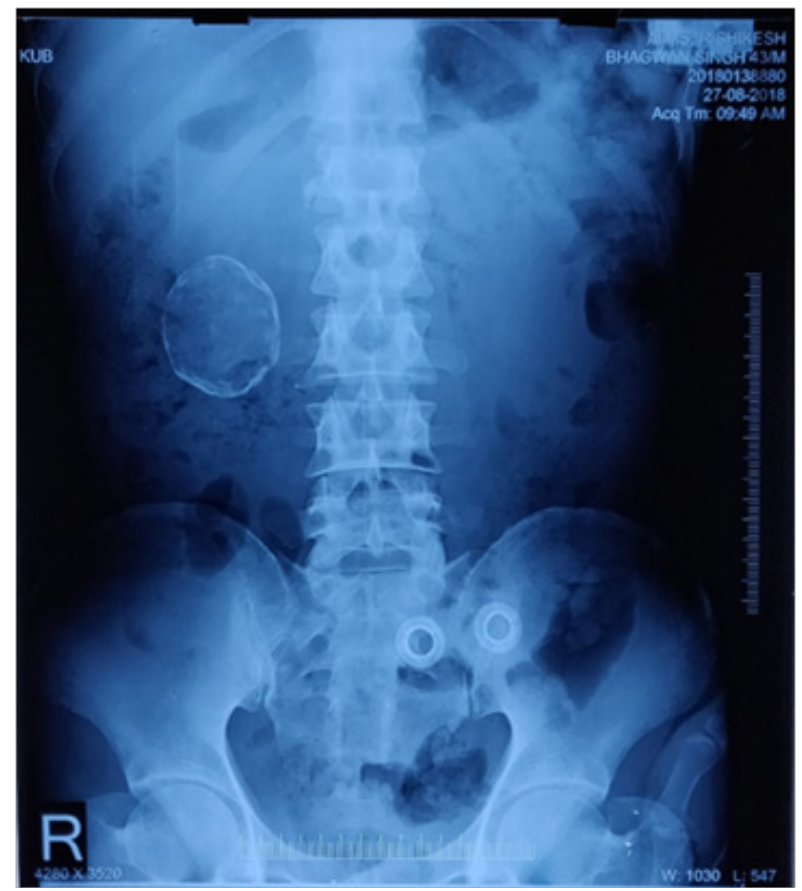

At the time of initial presentation his Random blood sugar values was $468 \mathrm{mg} / \mathrm{dL}$.Other routine tests tests were within normal limits. His serum amylase levels were $185 \mathrm{U} / \mathrm{L}$ and serum Lipase levels were 467 U/L. Furthermore, urinary ketone bodies were negative and Arterial blood gas analysis was not suggestive of any high anion gap metabolic acidosis. Stool examination revealed elevated fat content on examination. An AP X-ray radiogram of the abdomen showed a calcified cystic lesion to the right of the midline at the level of L2/3 vertebral bodies.

A CECT abdomen was done which showed extensive calcification and atrophy of the pan-creas with a $6.5 * 6.8 * 7.6 \mathrm{~cm}$ pseudo cyst involving the pancreatic head. There were similar but smaller pseudo cysts involving the body and tail of the pancreas.Multiple other calcific foci were also found in various soft tissue locations .His serum C-peptide levels were found to be $0.11 \mathrm{ng} / \mathrm{ml}$.

Based on the above presentation he was diagnosed to have chronic calcific pancreatitis related diabetes. He was started on Inj. Glargine 8 units HS and inj. human insulin regular 4units Thrice a day before each meal.

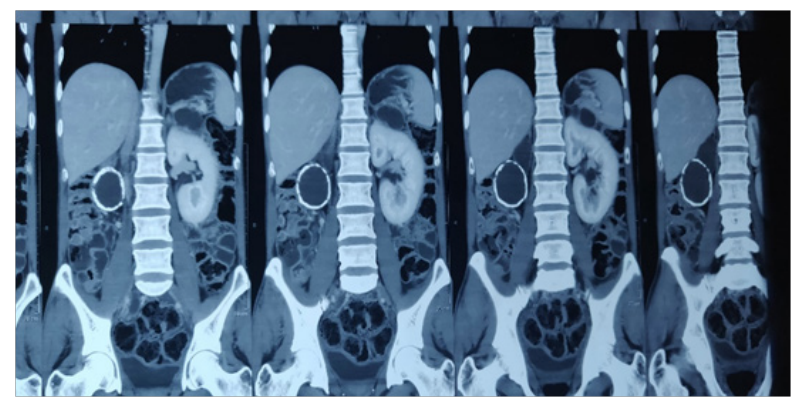

Figure 2: psuedocyst involving head and tail of pancreas

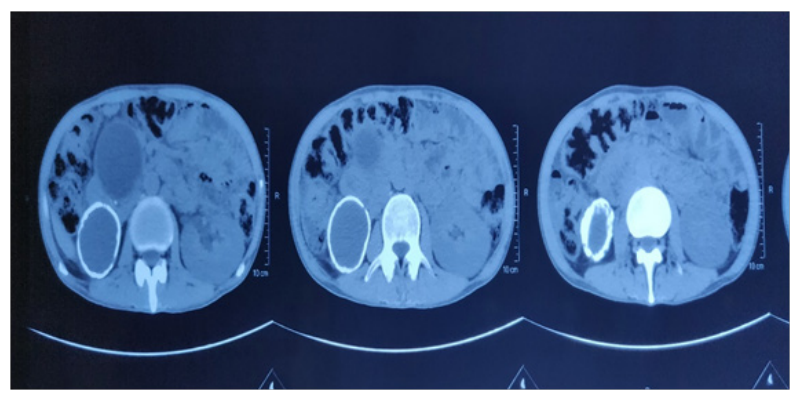

Figure 3: psuedocyst involving the head of pancreas showing calcification 


\section{Discussion}

Chronic pancreatitis is most commonly caused worldwide in adult patients by alcohol re-lated recurrent pancreatitis. In children the most common cause is cystic fibrosis. Presently the etiology of chronic pancreatitis is classified using the TIGAR-O classification (Toxic, Idiopathic, Genetic , Autoimmune, Recurrent and Obstructive ). In non-alcoholic young adults an important cause is tropical fibro-cacific pancreatitis which is relatively more common in the Indian states of kerala and Tamil Nadu . No cases have yet been documented from Uttarakhand. Autoimmune pancreatitis may occur with $\mathrm{IgG} 4$ related disease (type 1$)^{3}$ and as [part of autoimmune polyglandular syndrome type 1 and 2. Rarer genetic causes of chronic pancreatitis include patients with mutations in the SPINK1 (encoding trypsinogen inhibitor), CTRC(encoding chymotrypsin C ), PRSS1(cationic trypsinogen) and $\mathrm{CaSR}$ (calcium sensing receptor loss of function mutation). ${ }^{4,5}$

The etiology of tropical fibro-calcific pancreatitis is multifactorial and poorly understood with suspected causes being a genetic predisposition, selenium deficiency, cyanide toxicity secondary to chronic dietary ingestion. Criteria for diagnosis of tropical fibro calcific pan-creatitis are as follows 1)Occurring in a "tropical" country 2) established Diabetes mellitus based on ADA criteria 3) radiographic evidence of chronic pancreatitis and 4) absence of other causes of chronic pancreatitis. ${ }^{6}$

The diabetes associated with FCPD is associated with decreased serum C-peptide levels (as seen in this patient) and is usually severe in magnitude. However, the incidence of di-abetic ketoacidosis is very low which is largely a result of the low body fat content of the patients secondary to malabsorption. There is also a lower incidence of the macro vascular complications of diabetes. But the microvascular complications such as neuropathy, nephropathy and retinopathy are common and related to long term glycemic control. ${ }^{7,8}$

The mechanisms for development of diabetes in chronic pancreatitis are 2 fold -primary destruction of beta cells and decreased intestinal increasing secretion as a result of malabsorption.

Multiple soft tissue calcifications is a relatively uncommon radiological finding with many plausible causes which may be remembered using the pneumonic TIC MTV ${ }^{9}$

T- Tumor ( Tumoral calcinosis, synovial osteochondromatosis , calcified lipoma/ hemangioma/sarcoma)

I- Inflammation (Systemic sclerosis, Dermatomyositis, pancreatitis with metastatic calcification, calcific myonecrosis, hydatid cysts )

C-congenital (Ehlers-Danlos syndrome, Myositis ossificans progressiva)

M-Metabolic (Hyperparathyroidism[primary/ secondary] ,calcium pyrophosphate deposition disease[CPPD], calcium hydroxyapatite deposition) T-Trauma ( Myositis ossificans, burns, hematoma) V-Vascular (chronic venous insufficiency, arterial calcification)

\section{Conclusion}

Chronic fibro calcific pancreatitis is a rare cause of diabetes in young patients but has a characteristic clinical picture with a young patient presenting with features of malabsorption and low BMI, low propensity for DKA, low beta cell reserve and sensitivity to Insulin. The etiology of chronic pancreatitis can be varied and occasionally multifactorial and therefore requires a detailed structured work up . Timely diagnosis, insulin supple-mentation and tight glycemic control is required to prevent the development of microvascular complications of diabetes .

\section{REFERENCES}

1) WHO Study Group Report on Diabetes Mellitus. WHO technical report series 727. Geneva: WHO; 1985.

2) Expert Committee on the Diagnosis and Classification of Diabetes Mellitus Report of the Expert Committee on the Diagnosis and Classification of Diabetes Mellitus. Diabetes 
Care

3) Kamisawa $\mathrm{T}$, Chari ST, Giday SA, Kim MH, Chung JB, Lee KT, Werner J, Bergmann F, Lerch MM, Mayerle J, et al. Clinical profile of autoimmune pancreatitis and its histological subtypes: an international multicenter survey. Pancreas. 2011;40:809-814.

4) Heiko Witt, Werner Luck, Hans Christian Hennies, Martin Claben, Andreas Kage, Ulrich Lab, Olfert Landt \& Michael Becker. Mutations in the gene encoding the serine protease inhibitor, Kazal type 1 are associat-ed with chronic pancreatitis.BMC Gastroenterol. 2003; 3: 34. 2003 Nov 29. doi: 10.1186/1471-230X3-34.

5) Balázs Csaba Németh $\mathrm{MD}, \mathrm{PhD}$, Ákos Szücs MD, PhD, Péter Hegyi MD, PhD, DSci \& Miklós Sahin-Tóth MD, PhD. Novel PRSS1 Mutation p.P17T Validates Pathogenic Relevance of CTRC-Mediated Processing of the Trypsinogen Activation Peptide in Chronic Pancreatitis.(Under Publication)
6) Whitcomb DC, Gorry MC, Preston RA, Furey W, Sossenheimer MJ, Ulrich CD, et al. Hereditary pancreatitis is caused by a mutation in the cationic trypsinogen gene. Nat Genet. 1996;14:141-5. doi: 10.1038/ng1096-141.

7) Mohan R, Rajendran B, Mohan V, Ramachandran A, Viswanathan M. Retinopathy in tropical pancreatic diabetes. Arch Ophthalmol. 1985;103:1487-9. doi: 10.1001/ archopht.1985.01050100063020.

8) Ramachandran A, Mohan V, Kumaravel $\mathrm{TS}$, Velmurugendran $\mathrm{CU}$, Snehalatha $\mathrm{C}$, Chinnikrishnudu M, et al. Peripheral neuropathy in tropical pancreatic diabetes. Acta Diab Lat. 1986;23:135-40. doi: 10.1007/BF02624673.

9) Banks KP, Bui-Mansfield LT, Chew FS etal. A compartmental approach to the radiographic evaluation of soft-tissue calcifications. Semin Roentgenol. 2005;40 (4): 391-407. doi:10.1053/j.ro.2005.01.021. 